\title{
New Members of Division 32
}

The following fifty-eight persons were accepted for membership in Division 32, effective January 1, 1989:

\section{Members}

Thomas L. Armstrong

Raymond F. Bakaitis

Fred Bemak

Daniel L. Boland

Alan L. Braunstein

William A. Carlson

Brant Cortright

Pamela Cotton

Michael B. DeMaria

Joyce M. Eckblad

Don E. Hamachek

Mufid J. Hannush

Patricia A. Hanson

Robert L. Harman

Ranni Haynes

David L. Hellkamp

Stephen Hoyer

Carl F. Hurwitz

Seyed M. Jalalt-Tehrani

John P. Keenan

Virginia A. Larson

Bruce L. Levine

Michael S. Lieberman

Larry M. Leitner
Denuth C. Lougeay

Maralyn Luber

Brent S. Mallinchrodt

Michael J. Murphy

Laura S. Morrison

Nancy Parsons-Craft

Herbert M. Potash

Jesse A. Rabinowitz

Donadrian L. Rice

William T. Scofield

Kenneth J. Shapiro

David L. Smith

Richard A. Sobel

Maryhelen Snyder

Michael J. Stevens

Edward M. Stodola

Robert Storms

William F. Tageson

L. Eugene Thomas

Duane L. Varble

Teddy D. Wamer

Paul T. P. Wong

Roger W. Woodbury

Jing-Jyi Wu

Associate Members

Ray M. Adomaitis

Christine E. Beal .

Deborah A. Draving

Edward H. Fankhanel

Rose Marie E. Gordon
Laurel A. Leland

Eduardo A. Lombard

Bradford S. Munger

Thomas A. Sherralt

Kenneth R. Vanderlip

Submitted by

Frederick J. Wertz, Division 32 Membership Chair

Vol. 16, No. 2, Autumn 1988 